\title{
Uso de substâncias e função sexual em adolescentes com doenças crônicas
}

\author{
Acadêmicos: Márcio Guilherme Nunes Carvalho, Priscila Araújo, Marlon Van Weelden \\ Orientadores: Benito Lourenço, Lígia Bruni Queiroz, Clovis Artur Almeida da Silva
}

\begin{abstract}
Introdução: A adolescência é marcada por mudanças biológicas e sociais. Nesse período, comportamentos de risco podem ter início, destacando-se aqueles relacionados ao uso de substâncias e à atividade sexual. Observa-se um aumento da prevalência de doenças crônicas na população adolescente, o que se soma a outros agravos que afligem essa população. Dentre esses, aqueles relacionados aos comportamentos de risco mencionados merecem destaque por serem relativamente frequentes. Desse modo, justifica-se a realização de estudo que permita avaliar a ocorrência desses agravos e que dê ênfase à população adolescente com doenças crônicas (ADC's).
\end{abstract}

Objetivo: Avaliar o uso de álcool, tabaco e drogas ilícitas em adolescentes com e sem doenças crônicas. Avaliar possíveis associações entre o uso de substâncias por ADC's e os seguintes fatores: dados demográficos, desenvolvimento puberal e atividade sexual.

Metodologia: Estudo de corte transversal com 220 ADC's e 110 controles, consistindo na aplicação de questionário aos adolescentes (10-19 anos) ao término dos atendimentos ambulatoriais. Doenças crônicas foram definidas como tendo duração $\geq 3$ meses. O questionário foi aplicado mediante obtenção de consentimento verbal, em conversa privada com o paciente e incluiu: dados sócio-demográficos e antropométricos; avaliação do desenvolvimento puberal; versão em português do questionário CRAFFT, utilizado para rastreamento de risco aumentado para abuso/dependência de substâncias na adolescência; questões adicionais sobre uso de substâncias e função sexual. Realizou-se teste preliminar com 30 ADC's e controles para avaliação da confiabilidade do questionário, tendo o teste sido repetido um e dois meses depois. A análise estatística incluiu: cálculo do índice Kappa; testes t ou Mann-Whitney; testes exato de Fisher ou Qui-Quadrado; Coeficiente de correlação de Spearman. Nível de significância: 5\%.

Resultados: O índice Kappa foi de 0.850. Adolescentes com e sem doenças crônicas apresentarem valores similares na mediana da idade atual [14 (10-18) vs. $14(10-18), p=0.778)$ e na frequencia de uso de substâncias lícitas e/ou ilícitas ( $30 \%$ vs. $34 \%, \mathrm{p}=0529)$.

Dentre os que apresentam doenças crônicas, os que declararam já ter feito uso de substâncias apresentam valores superiores de: mediana da idade atual [15 (11-18) vs. 14 (10-18), $\mathrm{p}<0.0001$ ]; frequências de Tanner 5 ( $<<0.0001)$ e de atividade sexual $(23 \%$ vs. $3 \%, p<0.0001)$. Uma correlação positiva foi observada entre a pontuação no CRAFFT e a idade atual em ADC's $(\mathrm{p}=0.005, \mathrm{r}=+0.189)$.

Discussão: O valor de Kappa obtido indica excelente confiabilidade para o questionário utilizado. Dentre os ADC's, a mediana de idade mais elevada apresentada pelo subgrupo que refere uso de substâncias pode estar relacionada à ocorrência de uma vigilância parental mais intensa no início da adolescência, que pode se afrouxar posteriormente. A maior frequencia de atividade sexual verificada nesse subgrupo pode estar relacionada a uma maior maturação sexual devido à idade mais avançada. A correlação positiva entre a pontuação no CRAFFT e a idade atual em ADC's sugere risco aumentado para uso nocivo de substâncias em indivíduos mais velhos. O estudo reforça a importância de, nas consultas pediátricas, ADC's com histórico de uso de substâncias passarem por rastreamento para abuso de substâncias e atividade sexual de risco, especialmente ADC's mais velhos. 